\title{
Sensibiliser à l'environnement avec des animaux empaillés : Les trophées du Musée de la Chasse et de la Nature
}

\section{Antoine Jeanne}

\section{(2) OpenEdition}

\section{Journals}

Édition électronique

URL : https://journals.openedition.org/ere/4480

DOI : $10.4000 /$ ere.4480

ISSN : 2561-2271

Éditeur

Centr'ERE

\section{Référence électronique}

Antoine Jeanne, "Sensibiliser à l'environnement avec des animaux empaillés : Les trophées du Musée de la Chasse et de la Nature », Éducation relative à l'environnement [En ligne], Volume 15 - 1 | 2019, mis en ligne le 20 décembre 2019, consulté le 15 octobre 2021. URL : http://journals.openedition.org/ere/ 4480 ; DOI : https://doi.org/10.4000/ere.4480

Ce document a été généré automatiquement le 15 octobre 2021

\section{(c) (†) 8}

La revue Éducation relative à l'environnement est mise à disposition selon les termes de la Licence Creative Commons Attribution - Pas d'Utilisation Commerciale 4.0 International. 


\title{
Sensibiliser à l'environnement avec des animaux empaillés : Les trophées du Musée de la Chasse et de la Nature
}

\author{
Antoine Jeanne
}

1 Exhiber l'animal mort ne date pas d'hier. À travers les âges, et par-delà les contextes anthropologiques, les restes cynégétiques revêtent des formes et des usages aussi divers que variés. Il demeure cependant un invariant: la dépouille suppose une dialectique entre nature et culture. Conserver puis exposer la carcasse d'un animal chassé permet d'affirmer la victoire de l'Homme sur la nature, de revendiquer son appartenance à la culture.

2 Dès lors que l'on considère la maison comme l'endroit où l'on se cache de la nature, les murs et le toit marquant la frontière symbolique et matérielle avec la culture, pourquoi voudrait-on la faire entrer par le biais d'une dépouille vidée de sa viande ? Comment expliquer qu'un musée, qui conserve ce qu'une société produit ou possède, de plus culturellement représentatif et significatif - de l'artefacta à l'exotica en passant par le scientifica ou le mirabilia - soustraie aux lois de la vie un être putrescible pour en faire un musealia. Quel(s) usage(s) un musée réserve-t-il à ce naturalia qui dépasse les frontières ontologiques $^{1}$ ?

3 Aussi, afin de traiter de l'éducation à l'environnement au sein d'un musée cynégétique, nous nous attarderons au Musée de la Chasse et de la Nature de Paris. Comment est-on passé d'un musée dominant, ancré dans une vision colonialiste et prédatrice de l'animal et de la nature, à un musée écologiste qui interroge notre rapport à la nature en (re)constituant un havre de paix au cœur du quartier du Marais? Quels effets la transformation d'un musée militant vers un musée poétique à la muséographie détonante a-t-elle eue sur la (re)présentation ainsi que sur la pédagogie de la nature? Et comment l'animal empaillé peut-il signifier autre chose que sa mort? 
4 Pour ce faire, nous suivrons l'évolution historique ainsi que le changement de paradigme muséal, Nous nous appuierons ensuite sur les outils de médiation mis en place par le Musée de la Chasse et de la Nature, notamment auprès des jeunes visiteurs, afin de rendre tangible le regard porté sur la nature. Puis nous aborderons l'utilisation paradoxale de taxidermies cynégétiques pour porter un message sensible sur la nature.

\section{Une musésographie détonnante}

5 À l'origine du Musée de la Chasse et de la Nature il y a un couple passionné par les animaux. François ${ }^{2}$ et Jacqueline ${ }^{3}$ Sommer consacrent toute leur vie à la chasse, à la nature, à la réintroduction d'espèces, à l'art animalier ou bien encore à la littérature cynégétique. Il est également une autre passion inhérente à la cynégétique: la collection. Elle est une alternative au tir. La possession d'objets relatifs à la chasse est une manière d'en prolonger le plaisir. On renouvelle l'émotion de la traque en s'entourant d'un décor composé de trophées et de différentes pièces d'art, d'armes, d'ouvrages littéraires ou bien d'objets ethnographiques. Cette collection constitua le noyau du futur Musée de la Chasse et de la Nature.

6 Les Sommer souhaitent mettre leur patrimoine au service de leurs engagements et créent la Fondation de la «Maison de la Chasse et de la Nature ${ }^{4}$. " Dépositaire de leur collection, celle-ci pérennise à la fois un legs et continue leur dessein. La Fondation devient rapidement un outil influent pour promouvoir la défense de la chasse, de la faune et de la nature. Elle compte deux avatars patrimoniaux majeurs: Le Parc de vision de Belval ${ }^{5}$, afin de faire découvrir le plaisir de la rencontre avec l'animal et de conserver un patrimoine naturel régional ; puis un musée cynégétique.

7 Le Musée de la Chasse et de la Nature est inauguré le 21 février 1967, soutenu dès 1964 par le Ministère des Affaires culturelles afin de faire consensus autour de la nature ${ }^{6}$. Face à la politique d'acquisition du musée, les bâtiments deviennent rapidement trop petits. L'hôtel de Mongelas fait alors office de favori pour agrandir le musée. La bâtisse du XVI ${ }^{\text {ème }}$ siècle est acquise le 18 avril 2002 permettant l'étude d'extension du musée ${ }^{7}$. Les travaux débutent le $1^{\mathrm{er}}$ janvier 2005 et s'étendent jusqu'au 6 juin 2007. Le Musée de la Chasse et de la Nature est d'abord le résultat du labeur des Sommer qui conçoivent un musée-maison, écrin pour leurs collections, et «l'hybridation entre un rendez-vous de chasse et un cabinet d'amateur " (Anthenaise, 2007, p. 56). Toutefois, entre le musée conçu par les Sommer dans les années 1960 et le musée incontournable des années 2000, il s'opère un véritable changement de paradigme muséologique.

8 La volonté muséale primaire est de témoigner de l'universalité de la chasse. Il est ardu d'exprimer l'expérience de la nature, de la faune sauvage et de leur nécessaire gestion cynégétique. Comment retranscrire cela? Comment prolonger des plaisirs éphémères ? Et surtout, comment traduire matériellement la nature, elle qui échappe à l'Homme?

9 Le discours adopté est résolument encyclopédique. La muséographie classifie les techniques de chasse et les animaux, procède à des regroupements géographiques et historiques, puis immerge le visiteur. Le parcours, ponctué d'œuvres d'art animalier ${ }^{8}$ et d'objets cynégétiques ${ }^{9}$, s'attache à donner aux visiteurs le charme d'une maison du Grand Siècle. Les Sommer pilotent le projet muséographique et la décoration se fait selon leurs goûts qui correspondent à l'esthétisme des années 1960. L'accrochage est profus et mélange les genres. Le modernisme côtoie l'historique. Fuyant le classicisme 
et la froideur muséale, ce musée-maison évoque le repos idéal du chasseur en juxtaposant pléthores d'objets, rappelant le goût des chasseurs pour la profusion des souvenirs cynégétiques ${ }^{10}$. Dans un premier temps, c'est une nature apprivoisée, anthropomorphe et ethnocentriste que le musée expose. Le chasseur domestique cette nature, organise et colonise le monde. La chasse est l'outil qui confronte l'Homme à la nature et à l'altérité, à sa connaissance et à son appréhension. La muséographie de « La salle d'Afrique " témoigne de la double domination sur l'animal et l'Homme (Dias, 1999), étendant ainsi ce que l'on peut trophéiser. Les objets ethnographiques sont exhibés au même titre que les animaux naturalisés ${ }^{11}$, sans réelle distinction spatiale ni conceptuelle. Implicitement, on indique que des populations vivant à l'état de nature méritent d'être exposées aux côtés de cadavres d'animaux. Cette violence symbolique véhicule l'idée que la prédation est un moyen légitime de muséaliser ${ }^{12}$.

Le musée, ainsi sanctuarisé durant une quarantaine d'années, perd doucement de son intérêt au regard des enjeux environnementaux du XXI ${ }^{\text {ème }}$ siècle. Il séduit davantage les chasseurs convaincus et n'attire guère les citadins coupés de la nature qu'il voulait éduquer aux principes cynégétiques. Il convient alors de repenser le discours relatif à la chasse, à la nature et à l'animal, face à une société qui se montre de plus en plus hostile quant aux pratiques cynégétiques ${ }^{13}$. La promotion cynégétique semble inappropriée à l'essor muséologique. Néanmoins, la critique fondamentale de la chasse ne permet pas non plus de positionner un musée sur une pratique qui est à l'origine de nos sociétés (Ortega Y Gasset, 1942) et dont le patrimoine témoigne largement (Beurdeley, 2004 et Chevée, 2018). Quant aux problématiques écologistes, elles sont devenues un fait social total, dans la mesure où elles mobilisent l'ensemble de la société. Un musée ne peut plus agir désormais sans avoir la moindre considération réflexive et référentielle sur ces notions. C'est dans ce contexte que des travaux de rénovation - architecturales et muséographiques - sont entrepris, marquant ainsi l'évolution paradigmatique du musée.

11 Renouvellement muséographique ne rime toutefois pas avec déperdition d'âme d'un lieu. Le charme du Musée de la Chasse et de la Nature opère dans l'illusion de visiter une maison particulière. Aussi la nouvelle muséographie s'attache-t-elle à conserver l'aspect de demeure en adoptant une scénographie affective et intimiste. Celle-ci juxtapose les musealias, sans lien apparent les uns avec les autres, sans devoir justifier d'une quelconque pertinence sémantique. Cela fait sens dans une collection particulière où le goût, l'envie et la décoration priment sur le discours. Les fauteuils ou les canapés renforcent cette impression. Le visiteur est invité à prendre son temps, comme dans un salon. Il aborde plus librement les œuvres, puisque les collections ne nécessitent aucune bonne manière ou initiation (Bourdieu, 1966), elles s'offrent à qui veut les admirer. Pour renforcer l'impression de vieille demeure, le musée multiplie les références aux Sommer, comme si nous étions chez eux. Ainsi la visite débute par le sanglier ${ }^{14}$, pour se conclure par l'évocation du relais de chasse ardennais du couple, comme si par-delà les fenêtres, nous n'étions plus dans le quartier du Marais à Paris. Car si les références et les artifices pour faire de ce musée une demeure particulière sont nombreux, ils sous-tendent néanmoins le véritable dessein scénographique du musée : celui d'une visite en forêt. Le parquet craquant au rythme des pas invite certes au calme et au respect des lieux, mais il incite surtout à déambuler discrètement et prudemment, comme si le sol était jonché de branches tombées des arbres et prêtes à craquer pour informer les animaux dont le visiteur se sait être à l'affût. Les animaux figurés vivants, grâce aux taxidermies en pied ${ }^{15}$, qui peuplent le musée peuvent 
disparaître à tout moment dans les profondeurs boisées. À force de métonymie et d'allégorie ${ }^{16}$, entre ville et forêt, le Musée de la Chasse et de la Nature se situe désormais hors de l'espace et du temps. La réflexion se dessine autour d'une nature surnaturelle que le musée s'efforce de mettre en mouvement à travers ses collections. Pour ce faire, le musée dérobe les conventions muséographiques, scientifiques et même cynégétiques. Cela lui permet de tirer profit d'objets initialement lourds de sens. Ceuxci ne sont alors plus la priorité puisque le musée s'oriente vers une muséologie d'idée (Mairesse, 2015). Ce détournement du musealia permet au Musée de la Chasse et de la Nature d'être un espace novateur et expérimental, propice à la réflexion sur la nature. Une nature, certes particulière, mais qui questionne l'évolution de la relation Hommeanimal, et sur le rôle de la chasse dans ce processus relationnel.

Aussi, dans cette nouvelle scénographie, l'ancienne "Salle d'Afrique " s'intitule-t-elle désormais la "Salle des Trophées» et concentre l'ensemble des montages en cape ${ }^{17}$. Il s'agit, encore une fois, d'un détournement muséographique, puisque la présentation sérielle des animaux mobilise explicitement les références organisationnelles des muséums d'histoire naturelle du XIX ${ }^{\text {ème }}$ siècle. Néanmoins, l'apparente taxinomie visuelle qui s'en dégage table sur un encyclopédisme vain. La série, dans sa mise en espace, ne fait qu'apporter un supplément esthétique aux objets (Bonnot, 2002). Ceux-ci font alors corps ensemble (Dassié, 2010). Du reste, les capes présentent un certain avantage muséographique : le gain de place. On peut donc observer et comparer un plus grand nombre d'animaux. À travers cette salle, le musée porte un regard sur la faune extra-euroépenne, sans toutefois prétendre à une quelconque exhaustivité. Il y est question d'un ailleurs et non plus d'une altérité. Rassembler les trophées permet non seulement d'établir une salle des trophées, comme pourrait le faire un chasseur, sans faire étalage de ces animaux dans l'ensemble du musée, mais surtout de faire le lien entre l'animal tropheíisé et son alter ego vivant à l'état de nature, qu'il convient de protéger. On voit donc poindre le discours environnementaliste, sans occulter l'origine de ces animaux puisque la salle est dite "des trophées. » L'Homme y prédomine à travers les fusils exposés en contre-bas, qui rappellent la victoire prédatrice sur la nature. Du reste, cela suggère que les trophées de chasse, à l'instar de beaucoup d'autres musealias, ne peuvent fonctionner en autonomie. C'est une réalité muséographique qui s'applique à la cynégétique: collections artistiques, ethnographiques et naturalistes se croisent et se confondent allègrement (Dumarteau, 2015). C'est un véritable accrochage à l'italienne ${ }^{18}$. Le Musée de la Chasse et de la Nature n'échappe pas à cette règle et nulle visite ne peut ignorer une typologie d'objets, car elles fonctionnent de concert. La collection consiste à rassembler des objets autour d'une étiquette commune et qui font sens ensemble. Aussi le mélange des genres et des typologies est-il admis, car c'est l'espace (muséal) qui va définir la relation entre des objets a priori distincts les uns des autres (Baudrillard, 1968). Il est même encouragé afin de renforcer l'impression de musée-maison ou de nature-boisée, dans laquelle les éléments cohabitent afin de soutenir une idéologie.

13 Le Musée de la Chasse et de la Nature transforme la cynégétique, mortifère en apparence, en concept muséologique, patrimonial, artistique, anthropologique, écologique et philosophique. Il s'impose désormais comme média (im)pertinent et légitime pour animer l'interaction entre l'Homme, l'animal et la nature. 


\section{Une médiation idéelle}

à l'environnement, aidé de ses trophées, il convient de se demander pourquoi mobiliser ces problématiques dans un musée (Chaumier et Porcedda, 2011)? En tant qu'institution majeure de notre société, un musée se doit d'être un acteur de la contemporanéité, en lien avec d'autres institutions, afin de répondre aux enjeux qui leur incombent. En l'occurrence, les réflexions environnementales étant au cœur de l'agora, le Musée de la Chasse et de la Nature les a intégrées.

15 Les prises de conscience environnementale s'égrènent depuis la Révolution industrielle, où certains esthètes et romantiques s'inquiètent du bouleversement paysager de l'Europe, jusqu'en 1971, où le Club de Rome alerte sur l'impact des agissements humains sur l'environnement. Entre temps, la Seconde guerre mondiale éveille les esprits puisque les milliers de bombes larguées perturbèrent des écosystèmes entiers, et perturbent encore à ce jour jusqu'à l'ionosphère (Major et Scott, 2018). La fin $\mathrm{du} \mathrm{XX}^{\text {ème }}$ siècle voit se multiplier les commissions visant à faire de l'environnement le sujet incontournable de ce début de XXI ${ }^{\text {ème }}$ siècle, où la société civile et la jeunesse s'emparent du sujet afin de faire pression sur les décisionnaires. Ainsi devenue un fait social total, l'écologie influe-t-elle sur différentes institutions. L'Éducation nationale en France suit de près ces évolutions. Entre les années 1970 et 2000, elle intègre progressivement des contenus pédagogiques relatifs à l'environnement: en 1971, le tout jeune ministère de l'Environnement incite les enseignants à promouvoir l'éducation à l'environnement (Poujade, 1975) ${ }^{19}$, et la circulaire du 15 juillet 2004 généralise l'éducation pour un développement durable ${ }^{20}$. coupe de Hugues de Varine, s'attachent à conserver des territoires et leur patrimoine immatériel dans une perspective d'écologie naturelle et d'écologie humaine, ou si dès 1967, les Parcs naturels régionaux se donnent pour mission de protéger et valoriser le patrimoine naturel, il faut attendre les années 1990-2000 pour que les musées pensent l'écologie comme un objet culturel et muséal. Il est difficile en effet pour ces derniers d'intégrer pleinement les concepts écologiques dans leurs pratiques muséographiques, davantage orientées vers la conservation plutôt que vers la prospective de sociétés dont ils se font les témoins et non les acteurs. L'environnement fait appel à un virage muséologique. On passe du musée-temps au musée-forum qui suscite le débat, la réflexion, la mobilisation et la responsabilisation (Davallon, Grandmont et Schiele, 1992). Du reste, la rencontre entre musée et écologie se suture lors du Sommet de la Terre de Johannesburg, tenu en 2002, qui propose de faire des musées des espaces d'expériences interdisciplinaires et de responsabilisation face aux enjeux environnementaux contemporains.

17 C'est dans ce paysage muséologique effervescent que le Musée de la Chasse et de la Nature met un terme en 2005 à sa désuétude muséale. Deux ans de refonte(s) plus tard, sous l'inspiration d'anthropologues, de philosophes, de conservateurs du patrimoine, de scientifiques et d'artistes, et s'étalant désormais sur $788 \mathrm{~m} 2$, le musée délaisse la promotion cynégétique pour inviter le visiteur à questionner la place de l'Homme dans la nature à travers le prisme de la chasse. Sous l'égide de la Fondation François Sommer pour la Chasse et la Nature, le musée fonctionne de pair avec l'École et Domaine de Belval afin de conduire des missions de protection et d'éducation relatives à la faune et 
à la flore. Quant à l'entité muséale, elle entend bien œuvrer pour le patrimoine artistique et culturel de la chasse. Dès lors, comment une muséographie cynégétique peut-elle accompagner des recommandations ministérielles?

Un premier élément de réponse se trouve dans la genèse du musée. Pour les Sommer, la chasse est un outil de régulation et de protection de la nature, perçue comme un patrimoine qu'il convient de conserver. Ils étaient convaincus du lien qui unit la gestion cynégétique à la sylviculture. Aussi François Sommer œuvra-t-il pour la création d'un Plan de chasse en 1963, d'un ministère de l'Environnement en 1971 et d'un examen théorique lors de l'octroi d'un permis de chasse en 1974 (Chemel, 2013). La chasse devait être au centre des politiques ruralistes et environnementalistes. Leurs collections personnelles devaient alors témoigner de l'aspect protecteur du chasseur. Car la chasse préserve des espaces naturels, maintient l'équilibre des espèces et génère un patrimoine culturel et naturel qui participe de notre société.

Le musée tint ensuite un discours historico-technico-cynégétique dont la propagande n'en fit pas un musée séduisant. La nouvelle muséographie prend à bras-le-corps la question environnementale en délestant la dichotomie musée scientifique versus musée de beaux-arts. Pour ce faire, le Musée de la Chasse et de la Nature opère une synthèse entre les arts et les sciences (humaines) afin de penser l'environnement à travers la lunette de la prédation. Dans ce nouveau parcours, le musée privilégie une approche émotionnelle plutôt que pédagogique (D'Anthenaise, 2009). En effet, le musée n'est pas une école. Si la pédagogie reste en filigrane du parcours, l'imaginaire et l'allégorie soutiennent davantage la muséographie. Du reste, au Musée de la Chasse et de la Nature, il faut prédater la connaissance. Rien n'est donné aisément dans cette scénographie exigeante, tout doit se chercher. Le visiteur est à l'affût du moindre animal, du moindre détail artistique ou du moindre pied de nez muséographique. L'appréhension est ludique, la déambulation est un plaisir. Ainsi, selon le temps, l'énergie voire le background dont dispose un visiteur, il pourra plus ou moins percer le discours muséal.

Le Musée de la Chasse et de la Nature se veut pudique. Il respecte les opinions, et le consensus ne figure pas parmi ses objectifs. Sans prétendre apporter de réponse, il invite subtilement le visiteur à ( $\mathrm{s}^{\prime}$ )interroger. Les musealias sont le support de sa réflexion, pas la réponse du musée, encore moins des fétiches. Ils concrétisent la réflexion, mais laissent la conclusion ouverte au dialogue. À travers l'histoire de l'art et l'histoire naturelle, le musée confronte un point de vue rationnel à un point de vue subjectif. La rêverie, la métamorphose et l'imaginaire se mêlent à l'observation, à la rigueur et à la connaissance. Animal, (non)chasseur et chimères se partagent cette forêt urbaine du Marais dans laquelle la connaissance se construit intimement en suivant une piste. Le dogme n'est nullement au bout de cette voie.

Le musée propose les axes de recherche qui constituent sa muséographie. Tout un chacun peut apprécier les œuvres et faire sien le raisonnement du musée, voire le prolonger. Le musée multiplie d'ailleurs les théatres de diffusion culturelle. Pour commencer, les salles d'exposition sont ouvertes à tous les publics. Des cabinets naturalistes, au nombre de trois, y sont à disposition afin de ponctuer le parcours d'informations encyclopédiques, naturalistes et mythologiques sur certains animaux. Cela concerne le sanglier, le cerf et le loup - et même une famille de lièvres, encimaisée depuis quelques années. On peut alors découvrir ces informations en parallèle d'une vidéo de l'animal dans son environnement naturel, à condition d'avoir l'œil et la 
patience, car nul animal ne se livre docilement. Ces cabinets possèdent en outre un tiroir où l'on peut observer les arses $^{21}$, pour le plus grand plaisir des petits, ainsi que les traces $^{22}$. De plus, les œuvres sont commentées dans des cartels généraux qui rassemblent salle par salle - afin de ne pas inonder de multiples cartels le parcours - les informations nécessaires (et contingentes). Libre à chacun de découvrir ce qu'il veut, comme il le veut, s'il le veut. Si besoin est, les agents de sécurité n'hésitent pas à y aller de leurs anecdotes pour ponctuer la visite de détails inattendus ${ }^{23}$. Conjointement au parcours permanent, les expositions temporaires mettent un artiste ou un sujet en lumière, toujours dans le but d'accroitre les réflexions muséales. Ces expositions chamboulent avec malice la muséographie permanente afin de renouveler l'observation du visiteur. En effet, si le musée dispose bel et bien d'un espace dédié aux expositions temporaires, celui-ci contient rarement en ses cimaises la totalité des oeuvres qui les composent. Le musée se (re)découvre dans son ensemble, incitant au rapprochement entre les collections et les thématiques. Quant à sa réflexivité, les actions culturelles du Musée de la Chasse et de la Nature ne manquent pas de la stimuler tout au long des saisons. Les conférences et les colloques, les salons littéraires et les lectures, les projections et les concerts ou bien encore les performances et les nocturnes, tout est mis en place pour que le patrimoine cynégétique mette en branle les axes de recherche du musée parisien à l'instar de l'écologie.

\section{Une pédagogie muséale spécifique}

Le Musée de la Chasse et de la Nature propose plusieurs grilles de lecture à ceux qui s'y aventurent. Ce n'est pas «le Musée du Bouquet de fleurs ici ${ }^{24}$.» Il faut donc attirer et contenter le plus possible de visiteurs avec un sujet brûlant. Chasseurs, opposants et néophytes doivent pouvoir partager cet espace apaisé, dont les collections s'offrent à tous. Il doit également être abordable par les enfants. Ces derniers représentent environ $20 \%$ des entrées ${ }^{25}$. Aussi convient-il d'adapter le musée aux enfants, mais pas seulement. Le musée propose une visite qui séduira également les adultes qui les accompagnent afin que tout le monde y trouve son compte. Parents et enfants doivent, idéalement, repartir en ayant pris du plaisir et découvert les collections (Jonchéry, 2008). Quant aux publics scolaires, le musée se doit de répondre au programme académique développé par l'enseignant(e) (Denaw, 2012). Cependant le musée n'entend pas scolariser son contenu. Il s'envisage davantage comme un espace singulier extrascolaire. L'apprentissage n'y est pas une fin en soi, cela dépend de la capacité du musée à divertir l'écolier en lui faisant oublier la routine scolaire afin de lui faire apprendre sans qu'il s'en rende compte. Le musée devient un instrument pédagogique annexe, qui fait le lien avec l'école (Coppey et Jacobi, 1995). Ainsi les collections muséales peuventelles servir le propos scolaire, en complétant un apprentissage, sans être une école (Allard et Boucher, 1991). Il faut toutefois veiller à ne pas instrumentaliser le musée. Si l'école vient au musée, ce n'est pas pour que le musée fasse école. C'est parce que le musée fait écho à l'école que l'on s'y rend, parce que son discours complète et optimise un apprentissage. C'est une méthode didactique en trois temps dans laquelle le musée intervient puisqu'on prépare, on suit et on analyse une visite à l'aide d'une leçon. Non formel et hors cadre scolaire, le musée devient alors un espace de diffusion de la Culture et de connaissances qui ne se substitue pas à l'école (Denauw, 2012). 
Néanmoins, son approche sensible et réflexive peut permettre d'intégrer des notions parfois trop abstraites et conceptuelles pour des enfants (Allard et Boucher, 1998).

Le service des publics du Musée de la Chasse et de la Nature conçoit lui-même la trame des visites proposées aux publics enfantins. Afin de saisir l'usage médiatique du trophée de chasse dans un dessein de sensibilisation et d'éducation à l'environnement, nous avons suivi et analysé trois visites différentes, proposées à des groupes scolaires. "Les chiens de chasse», "Le mythe de Diane» et «Le cabinet de curiosités " constituent notre corpus de médiation. Les visites guidées se déroulent selon une thématique bien précise, à la demande d'une classe ou bien sur proposition du musée, et racontent une histoire. C'est un fil narratif qui conduit les enfants à travers les salles du musée pour découvrir les œuvres qui permettent d'ancrer un discours autour de la nature ou de l'animal. Le musée propose alors un complément, un regard détourné sur ces notions, dans le but d'aider un(e) enseignant(e) à presenter aux enfants des concepts naturalistes et environnementaux, en lien avec un cours de Sciences et Vie de la Terre, en vue de les sensibiliser aux enjeux contemporains auxquels ils seront confrontés. C'est un parti pris sur l'appréhension spatiale du musée et de ses collections puisque le guide mène directement aux oeuvres, majeures ou confidentielles, afin d'aborder les concepts importants. Du reste, cela permet de s'éviter la fatigue muséale due à la première visite d'un lieu rempli d'une multitude d'objets, a fortiori si ce sentiment peut être exacerbé par un regard juvénile. Ainsi le parcours à contrecourant, narré par le fil rouge thématisé, est-il un biais original et pertinent pour rendre tangible le sous-texte des musealias, les raisons pour lesquelles on patrimonialise, rassemble et expographie des artefacts relatifs à une pratique de plus en plus dépréciée (Charollois, 2013 et Denhez, 2014). La réflexion porte sur les relations qui lient les musealias. Pêle-mêle on y retrouve la nature, la culture, l'animal(ité), le sauvage, l'humanité, l'Homme et la chasse. On questionne donc les frontières entre ces concepts, leur pratique, leur nécessité, leur paradoxe. On envisage alors l'environnement, l'écologie, la biologie, l'anthropocène ou la mythologie. On anticipe la morale à retenir.

Pour arriver à ces concepts, le Musée de la Chasse et de la Nature soulève des questions pragmatiques. Parmi elles, citons la domestication ou la cynégétisation de l'animal, les pratiques de chasse ou bien les usages des restes cynégétiques (de la consommation carnée jusqu'à la naturalisation). On questionne la relation prédatrice entre les vivants, quels qu'ils soient, et leur nécessité biologique, à l'aide des œuvres d'art qui représentent ou symbolisent une scène de chasse ${ }^{26}$. Quant aux trophées, ils permettent d'aborder la protection des animaux et la différence entre la chasse et le braconnage, à l'aide de l'émotion et du réalisme des animaux naturalisés. Cela n'a rien de partisan, il s'agit de (faire) réfléchir à l'éthique cynégétique pour penser plus amplement notre rapport à l'animal et au monde. On étudie également le biotope des animaux, leur régulation voire leur façonnage par l'Homme ou bien des récits de chasse. Le prisme de la prédation - voire l'allégorie de la prédation puisque les visiteurs sont à l'affût (Van Der Meersch, 2008) - permet de croiser l'histoire de l'art, l'histoire naturelle, l'anthropologie et la mythologie, à l'instar d'un braconnage disciplinaire qui accroît la connaissance (De Certeau, 1990). Alors, l'enchevêtrement des typologies d'objets conduit à des réflexions cosmogoniques sur l'origine et le fonctionnement du monde qui induisent les problématiques environnementales. Le Musée de la Chasse et de la Nature n'est pas un muséum d'histoire naturelle, un centre d'interprétation ou un parc naturel régional. Il ne le revendique d'ailleurs pas, mais il offre une vision alternative à 
l'éducation à la nature. Le musée suggère la visite d'une nature qui n'est pas (seulement) une réalité biologique, mais un concept façonné par l'art, l'histoire, la mythologie et la cynégétique. Il nous invite à regarder un monde onirique afin d'appréhender l'extérieur du musée et de cohabiter dans un univers commun avec les animaux. Or la chasse permet cette poésie de la nature (Genevoix, 1938).

\section{Le recours aux trophées}

Chacune des trois visites observées s'est achevée dans la « Salle des Trophées. » Le pari consiste à user d'animaux largement amputés et sciemment abattus, pour discourir sur le vivant. Et la tâche n'est guère simple dans la mesure où pour un néophyte, le trophée renvoie à tout ce que la chasse a de négatif: la mort et l'emprise de l'Homme sur l'animal pour son bon plaisir. Les trophées sont des cadavres qui tendent à rapprocher le chasseur de la figure du barbare (Dieudonné, 2018). Dès lors, comment mobiliser de tels musealias pour mener une visite allégorique du vivant? Comment sensibiliser à la nature en exhibant des animaux qui semblent a priori avoir manqué de l'empathie du tireur?

Du chien de famille gardé en souvenir au spécimen rare ou disparu exposé au Muséum national d'Histoire naturelle en passant par la dépouille détournée par l'art (contemporain), il existe pléthore de raisons et de manières de naturaliser l'animal. Exhiber l'animal mort prend une tournure muséographique avec l'apparition du Kunst und Wunderkammer. On ne conserve plus seulement une dépouille pour parer et protéger sa maison, ni pour témoigner d'un statut social. On l'expose. On la donne à voir à autrui pour signifier autre chose qu'elle-même. Du cabinet de curiosités jusqu'à la Nouvelle muséologie ${ }^{27}$, le statut de l'animal chosifié et patrimonialisé évolue à maintes reprises, questionnant la prédation comme outil de collecte (Bondaz, 2014) et pointant le paradoxe de l'illustration du vivant à travers le mort.

Les notions de biodiversitée $e^{28}$ et de développement durable ${ }^{29}$, datant respectivement de 1986 et 1987, sont postérieures à la création du Musée de la Chasse et de la Nature. Néanmoins, elles ont été complètement intégrées au nouveau parcours et le musée mobilise ses collections cynégétiques pour les appréhender. En effet, à mi-chemin entre la demeure idéale du chasseur et de la forêt giboyeuse, mais surtout à travers sa collection de trophées, le Musée de la Chasse et de la Nature propose une incursion dans la biodiversité en montrant les relations prédatrices entre les êtres qui composent le vivant afin, notamment, de prendre conscience de la fragilité de la nature et du nécessaire rôle protecteur qu'il convient d'adopter. L'ensemble des collections, artistiques et naturalistes, introduit l'idée d'interactions avec une nature qui n'est pas infinie et inépuisable, puisque mortelle. Les collections cynégétiques sont lourdes de sens. Le Musée de la Chasse et de la Nature prend ses responsabilités en faisant de ses trophées les représentants du patrimoine naturel pour opérer un changement de mentalité. Ainsi les trophées servent-ils à évoquer la Convention de Washington en 1973, l'Évolutionnisme ou bien la coexistence entre l'Homme, l'animal et la nature. Ce nouveau paradigme muséal invite à voir au-delà des trophées. Il envisage conjointement le patrimoine naturel et le patrimoine culturel.

Il existe deux typologies majeures de muséographisation de l'animal mort. D'un côté se trouvent les muséums d'histoire naturelle, dont le développement épouse les formes des évolutions paradigmatiques de la science et de la muséologie. Ils exposent des 
animaux naturalisés dans un but scientifique : l'on parle de spécimen. Ces taxidermies illustrent des concepts biologiques, écologiques, évolutionnistes, phylogénétiques ou éthologiques. Leur muséalisation va dans ce sens. De l'autre, les musées cynégétiques sont inspirés des galeries de trophées et sont modelés par les écomusées. Ils se développent, initialement, dès les années 1930, afin de protéger et promouvoir une pratique critiquée autant que menacée ; puis ils accompagnent le développement des sciences humaines, à l'instar de l'ethnographie (Mairesse, 2015). Monographique et spécialisé, leur muséographie décloisonne les disciplines et multiplie les typologies de collections afin de problématiser la thématique chasseresse. Les nombreuses taxidermies accompagnent le discours cynégétique : on parle de trophée de chasse. Ces taxidermies illustrent des notions naturalistes, scientifiques, artistiques ou culturelles matérielles ou immatérielles - à travers le prisme de la relation prédatrice entre l'Homme et l'animal comme art de vivre et élément de culturation. Les trophées participent de cette logique et leur muséalisation assume d'offrir à la monstration une taxidermie dont la genèse relève du choix délibéré d'avoir logé une balle dans le poitrail d'un animal afin de le chosifier. Ces objets ont un statut muséal particulier. D’une nécessité absolue dans la muséographie cynégétique, le trophée demeure un patrimoine complexe et embarrassant: c'est une image que l'on ne veut pas voir (D'Anthenaise, 2012). Ainsi est-il devenu un objet de décorum, un accessoire scénographique, censé accompagner un discours scientifique (Viel, 2002), et le mode de vie (Niederer, 1984) cynégétique. Le trophée relève du musée imaginaire (Malraux, 1996) de la chasse, bien qu'il ne soit pas l'item le plus valorisé (Abrille, 2015). Aussi convient-il de trouver des fonctions muséales qui légitiment l'origine chasseresse et l'usage muséographique d'une dépouille animale. Dégagés de rite, d'étiquette et d'idéologie, les objets décoratifs bénéficient d'une plus grande liberté organisationnelle et fonctionnelle (Baudrillard, 1968). Cette liberté profite au Musée de la Chasse et de la Nature qui se débarrasse de la négativité de cet objet.

L'origine du trophée est guerrière et anthropomorphe. Il s'agit de la prise de possession puis de l'exhibition des symboles relatifs à une victoire. Ce sont les armes et les dépouilles des vaincus (Abrille, 2005). La pratique du trophée consiste à affirmer puis à commémorer sa victoire en assimilant concrètement le corps et l'esprit de l'adversaire (Descola, 2005). Trophéiser un animal revient également à marquer son emprise sur un être doué de vie afin d'en conserver la physionomie, voire l'individualité, afin de revendiquer une victoire. Pour ce faire, l'animal monté en trophée est naturalisé. Le chasseur, aidé du taxidermiste, pérennise un moment furtif : celui de la rencontre avec l'animal sauvage tant désiré, si ce n'est fantasmé. Cela sublime la dépouille, assume et revendique son origine cynégétique. Il s'agit d'un processus transformel qui met en scène la mise à mort, le dépeçage puis la conservation d'un animal initialement putréfiable. L'animal change d'ontologie : de naturalia, il devient artificialia.

Intrinsèquement, un trophée de chasse est un objet formel et matériel. N'importe quel animal, tout ou partie, peut relever du trophée, dès lors qu'il y a une revendication chasseresse de l'origine de la dépouille. Néanmoins, le principe même de trophée semble se concentrer autour d'un animal parcellisé. Le montage en cape canalise alors l'intérêt, cynégétique et néophyte, pour faire de la tête animale l'archétype du trophée. L'entièreté du corps n'intéresse guère le chasseur victorieux. Une fois mort, l'animal sauvage n'est plus et la venaison n'a que peu de valeur, si ce n'est alimentaire. A contrario la tête, qui est le centre névralgique de l'âme et de la force de l'animal, présente les attributs qui lui confèrent le statut de trophée qualitatif. Ce sont les armes 
du vaincu (Blüchel, 2014). Ainsi entendons-nous par trophée de chasse les têtes de cervidés ou de bovidés présentant des bois ou des cornes, celles des suidés, des ursidés, des canidés et des félidés présentant des dents (ANCGG, 2013). Le corps peut toutefois avoir de la valeur si l'on considère les peaux tannées des mammifères ou bien les plumes d'oiseaux. Ce sont les dépouilles du vaincu. D'autres parties du corps peuvent aussi avoir une valeur cynégétique si l'on examine les objets et les parures qui sont issus des animaux. Enfin, un trophée peut également être en pied. Ce n'est donc pas la forme qui détermine le trophée. À l'instar de l'objet ethnographique, le trophée de chasse se muséalise pour ses qualités extrinsèques et se définit par sa mobilité conceptuelle. L'utilisation muséographique d'un animal trophéisé se justifie par le fait qu'un trophée n'est pas une fin en soi. Un trophée revêt des considérations scientifiques (étude et suivi d'un cheptel et d'un biotope), historiques (domaniales ou affectives), ou folkloriques (les moeurs cynégétiques). C'est la raison pour laquelle le trophée occupe une place si importante dans la culture matérielle cynégétique. Si le trophée est muséalisé pour ses qualités documentaires, il est cependant muséographié pour ses qualités esthétiques, décoratives et mobilières: les taxidermies servent à valoriser les oeuvres muséales environnantes. Il n'a désormais plus d'incidence pratique, il est là uniquement pour signifier (Baudrillard, 1968). Aussi cela laisse-t-il à chaque musée cynégétique le soin d'attribuer au trophée l'usage qui lui sied. Ces typologies sont des chimères conceptuelles perméables, car un même trophée peut recouvrir plusieurs de ces fonctions à la fois, dans la mesure où un artefact peut aisément être déplacé symboliquement sur l'échelle des valeurs (Bonnot, 2002). Le trophée est un palimpseste muséographique sur lequel il n'est pas inconcevable de rédiger un manifeste pédagogique environnementaliste puisque l'animal mort est extrait de la nature que l'on veut expographier. Un musealia peut effectivement recouvrir divers usages selon l'évolution et les besoins du musée (Grognet, 2005). Il en est de même pour le trophée de chasse. Cela témoigne in fine, du statut de l'animal dans nos sociétés dans lesquelles, mort ou vif, il est un être dont l'Homme s'accapare la gestion, voire la création et les revendications (Baratay, 2003). Ainsi trouve-t-il sa place au Musée de la Chasse et de la Nature, car il est un sémaphore multiple, un objet prétexte (Hainard, 1984), qui permet de mobiliser des concepts anthropologiques et muséologiques, à l'instar de la vie et de la mort.

31 La mort peut être scandaleuse, mais elle est intrinsèquement liée à la vie. En ce sens elle mérite d'être expliquée et exposée. Elle constitue d'ailleurs un véritable sujet épistémologique et muséologique (Becker et Debary, 2012). En effet, même si les trophées de chasse sont des animaux chosifiés, ils n'en demeurent pas moins des dépouilles dont l'exposition représente une violence visuelle. D'aucuns considèrent que les musées cynégétiques muséographient la peur et la souffrance des animaux et que l'exposition de taxidermies ne devrait être réservée qu'aux muséums d'histoire naturelle dont le discours scientifique légitime cette monstration (Schäfer, 2015). Les visiteurs peuvent faire preuve d'empathie envers ceux qu'ils considèrent comme des victimes innocentes. Or l'animal ne doit pas être perçu comme un martyr. Si les émotions peuvent être bénéfiques à la réflexion, il convient néanmoins de ne pas braquer les publics. Aussi faut-il parfois faire preuve de censure préventive afin de vérifier la pertinence de l'exhibition de ces taxidermies délicates. En tant que lieu de pacification sociale, un musée ne peut exacerber des tensions relatives à un sujet polémique. Il ne faut toutefois pas négliger les vérités sur la pratique exposée et donc 
user de métaphore pour que le regard du visiteur puisse questionner le réel à la sortie du musée (Gonseth, 2012).

Si le musée ne veut pas passer pour un cimetière d'animaux, il faut qu'il réussisse la métamorphose de faire du trophée un artefact qui rend hommage à l'animal. Le Musée de la Chasse et de la Nature ne met donc pas l'accent sur l'acte cynégétique lui-même, mais sur la beauté animale afin de questionner la relation Homme-animal. Ainsi crée-til un équilibre entre esthétique et éthique pour que la muséographie fonctionne (Gorgus, 2012). C'est du reste la raison pour laquelle le terme de trophée n'est jamais délaissé au profit d'un autre et que la chasse reste le prisme principal duquel l'on contemple les taxidermies. Un musée cynégétique montre la manière dont l'Homme a transformé le monde vivant qui l'entoure : il expose une histoire naturelle de l'Homme et une histoire culturelle de la nature. C'est la tâche du muséographe (Bahuchet, 2002). Si les musées cynégétiques trouvent leur genèse dans une histoire biologique et anthropologique de la chasse, leur muséographie tend à thématiser l'art de vivre relatif à la chasse, du rôle de la chasse dans l'anthropocène et donc de la fabrication de la nature, ainsi que la place de la prédation dans l'art et le rapport à l'animal.

Ceci étant, le musée résout la question de la mort très simplement : l'animal n'y meurt jamais. Soit il est mort, soit il est figuré ou supposé vivant. Si le sang vient à couler, ce n'est pas de la main de l'Homme. Ou bien un chien accomplit le travail de l'Homme, ou bien deux animaux sauvages se battent ${ }^{30}$. La mise à mort, celle donnée par l'Homme n'a pas droit de regard. Le moment précis où le sang coule est un plaisir d'initié que l'on ne peut exposer aux néophytes. Lorsqu'il s'agit de taxidermie, a fortiori en pied, l'animal doué de mouvement, d'intention et d'expression est pourtant mort. On le sait. Les enfants aussi, ou alors ils demandent aux adultes de leur confirmer. Il n'est d'ailleurs nul besoin de tricher sur l'origine des animaux ou a contrario d'en faire une quelconque apologie cynégétique. Les taxidermies sont assumées pour ce qu'elles sont: des trophées de chasse.

\section{Conclusion}

Si l'évolution muséographique du Musée de la Chasse et de la Nature ne constitue pas une synecdoque muséologique, elle fait tout de même office de référence en la matière. En effet, le dessein environnementaliste permet de repenser et de dépoussiérer un

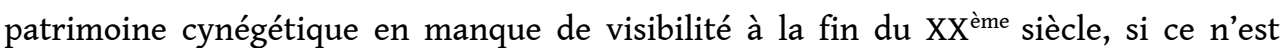
auprès des adeptes de Diane. Un usage muséographique parcimonieux des trophées, agrémenté d'une appréhension anthropologique, naturaliste et artistique permet au Musée de la Chasse et de la Nature la double prouesse de mobiliser un sujet clivant tout en composant avec un patrimoine jugé de mauvais goût il y a encore une décennie. Ainsi les animaux ne sont-ils pas morts une seconde fois.

La prédation n'est plus envisagée comme une fin en soi, mais comme un objet muséologique à part entière. La mort, nécessaire à la vie, n'y est donc pas encouragée ou magnifiée, mais seulement exposée et expliquée, comme le phénomène inhérent à la vie qu'elle est. Quant à l'animal, il demeure un sujet philosophique et anthropologique par-delà les contextes. Cependant, ce nouveau paradigme muséal persiste à désorganiser les frontières en faisant pénétrer la mort dans la sphère du vivant, et en faisant de l'animal une création humaine dont on fait des musealias. Pour justifier cela, les restes cynégétiques sont réifiés en hymnes à la vie. 
En ancrant ainsi un discours pédagogique et environnemental dans une dépouille animale, le Musée de la Chasse et de la Nature offre une caisse de résonance aux chasseurs. Pour ces derniers, l'utilisation de trophées de chasse n'a rien de paradoxal. En revanche, leur (ré)appropriation par les néophytes problématise la légitimité écologiste. Effectivement le chasseur, soutenu juridiquement et patrimonialement, s'affirme comme l'essence de l'écologie tandis que son antonyme, le très fantasmé citadin coupé des réalités rurales, continue de vouloir bannir la chasse. Gageons qu'à sa réouverture, prévue en octobre 2020 , le musée continuera d'être un espace pacifié où l'environnement se partagera, au même titre que son appréhension didactique.

\section{BIBLIOGRAPHIE}

Abrille, R. (2005). La mise en scène du sacrifice. Dans Conseil international de la chasse et de la conservation du gibier. Le chasseur et la mort (Actes du colloque « La chasse, une exception culturelle dans la vision contemporaine de la mort », pp. 111-142). Paris : La Table Ronde.

Abrille, R. (2015). Le musée (de chasse) imaginaire. Exposer la chasse ? Actes de colloque. (pp. 12-23). Paris : Musée de la Chasse et de la Nature. Consulté sur https://chassenature.org/wp-content/ uploads/2019/04/Actes-colloque-Exposer-la-chasse.pdf.

Allard, M. et Boucher, S. (1991). Le musée et l'école. Montréal : Éditions Hurtubise, Collection des cahiers du Québec.

Allard, M. et Boucher, S. (1998). Éduquer au musée : Un modèle théorique de pédagogie muséale. Montréal : Éditions Hurtubise, Collection des cahiers du Québec.

ANCGG (2013). Le grand gibier ; Les espèces, la chasse, la gestion. Paris : Gerfaut.

Bahuchet, S. (2002). L'homme indigeste ?. Dans M-O. Gonseth, J. Hainard et R. Kaehr (Dir.), Le musée cannibale (pp. 59-84). Neuchâtel : Musée d'Ethnographie de Neuchâtel.

Baratay, E. (2003). Et l'homme créa l'animal. Paris : Odile Jacob.

Baudrillard, J. (1968). Le système des objets. Paris : Gallimard.

Becker, A. et Debary, O. (2012). « Jochen Gerz et la parole maudite ». A. Becker et O. Debary (Dir.). Montrer les violences extrêmes (pp. 123-130). Grâne : Creaphis éditions.

Beurdeley, M. (2004). Pavillons de chasse. Saint-Rémy-en-L'Eau : Monelle Hayot Eds.

Blüchel, K. G. (2014). La Chasse. Paris : Ullmann.

Bourdieu, P. (1966). L'Amour de l'Art ; Les musées européens et leur public. Paris : Les Éditions de Minuit.

Bondaz, J. (2014). Entrer en collection; Pour une ethnographie des gestes et des techniques de collecte. Les Cahiers de l'École du Louvre, 4. Consulté sur https://journals.openedition.org/cel/481.

Bonnot, T. (2002). La vie des objets ; D'ustensiles banals à objets de collection. Paris : Maison des Sciences de l'Homme, Ethnologie de la France. 
Chaumier, S. et Porcedda, A. (2011). Musées et développement durable. Paris : La documentation française.

Charollois, G. (2013). Pour en finir avec la chasse ; La mort-loisir, un mal français. Paris : IMHO.

Chemel, F. (2013). François Sommer; Un temps d'avance. Paris : Buchet-Chastel.

Chevée, A. (2018). Art et chasse : Chassé-croisé. Paris : Gerfaut.

Coppey, O. et Jacobi, D. (1995). Introduction, musée et éducation : Au-delà du consensus, la recherche de partenariat. Publics et musée 7(7), 10-22. Consulté sur https://www.persee.fr/doc/ pumus_1164-5385_1995_num_7_1_1053.

Dalla Bernardina, S. (2013). Hymnes à la vie ? Sur l'engouement récent pour les bêtes naturalisées. Terrain, Revue d'ethnologie de l'Europe, "L'imaginaire écologique », 60, 56-73.

D’Anthenaise, C. (2007). Le Cabinet de Diane; Au Musée de la Chasse et de la Nature. Paris : Citadelles et Mazenod.

D’Anthenaise, C. (2009). À la recherche des visiteurs perdus. Les cahiers du musée des confluences, n 4, pp. 109-119.

D’Antenaise, C. (2012). L'image interdite. Billebaude. Paris : Glénat, n 1, pp. 60-65.

Dassié, V. (2010). Objets d'affection; Une ethnologie de l'intime. Paris : CTHS, Le regard de l'ethnologue, $\mathrm{N}^{\circ} 22$.

Davallon, J., Grandmont, G. et Schiele, B. (1992). L'environnement entre au musée. Lyon : Presses universitaires de Lyon.

De Certeau, M. (1990). L'invention du quotidien ; 1. arts de faire. Paris : Gallimard.

Denauw, C. (2012). Éducation et musée : un tandem complexe. Sociétés, 4, nº 118.

Denhez, F. (2014). La chasse; Le vrai du faux. Paris : Delachaux et Niestlé.

Descola, P. (2005). Par-delà nature et culture. Paris : Gallimard.

Dias, N. (1999). L'Afrique naturalisée. Cahiers d'études africaines, 39 (155-156), 583-594.

Dieudonné, A. (2018). La vraie vie. Paris : L’Iconoclaste.

Dumarteau, M-B. (2015). Muséifier la chasse : Charles-Jean Hallo, Aux origines d'un modèle muséographique. Exposer la chasse ? Actes de colloque (pp. 24-33). Paris : Musée de la Chasse et de la Nature. Consulté sur https://chassenature.org/wp-content/uploads/2019/04/Actes-colloqueExposer-la-chasse.pdf.

Genevoix, M. (1938). La dernière harde. Paris : Flammarion.

Gonseth, M-O. (2012). Montrer, montrer qu'on montre où montrer qu'on ne montre pas ; Les violences de guerre dans les expositions du MEN. Dans A. Becker et O. Debary (Dir.). Montrer les violences extrêmes (pp. 239-258). Grâne : Creaphis éditions.

Gorgus, N. (2012). Peut-on faire une belle exposition sur la guerre ?. Dans A. Becker et O. Debary (Dir.). Montrer les violences extrêmes (pp. 203-215). Grâne : Creaphis éditions.

Grognet, F. (2005). Objets de musée, n'avez-vous donc qu'une seule vie ?. Gradhiva, 2, 49-63.

Hainard, J. et Kaehr, R. (1984). Objets prétextes ; Objets manipulés. Neuchâtel : Musée d'Ethnographie de Neuchâtel.

Jonchery, A. (2008). Se rendre au musée en famille. La lettre de l'OCIM, 115, 4-14. 
Mairesse, F. (2015). Le paysage et l'avenir contrastés des musées de la chasse. Exposer la chasse? Actes de colloque (pp. 225-237). Paris : Musée de la Chasse et de la Nature. Consulté sur https:// chassenature.org/wp-content/uploads/2019/04/Actes-colloque-Exposer-la-chasse.pdf.

Malraux, A. (1996, 1ère Ed. 1965). Le musée imaginaire. Paris : Gallimard.

Niederer, A. (1984). Le langage des objets. Dans Hainard J. et Kaehr R., Objet prétextes; Objets manipulés (pp. 151-166). Neuchâtel : Musée d'Ethnographie de Neuchâtel.

Ortega Y Gasset, J. (1942). Méditations sur la chasse. Québec : Septentrion.

Poujade, R. (1975). Le ministère de l'impossible. Paris : Calmann-Lévy.

Scott, C-J. et Major, P. (2018). The ionospheric response over the UK to major bombing raids during World War II. Geophys, 36, 1243-1254.

Schäfer, V-A. (2015). Homme-Chasse-Animal ; Comment exposer la chasse à l'exemple du Musée de la Chasse et de la Nature à Paris. Mémoire d'Étude inédit, $1^{\text {ère }}$ année de $2^{\text {ème }}$ cycle. Paris : École du Louvre.

Soulages, F. (2012). Monstrations et expositions photographiques des violences. Dans A. Becker et O. Debary (Dir.), Montrer les violences extrêmes (pp. 45-60). Grâne : Creaphis éditions.

Van Der Meersch, C. (2008). Une visite sous forme d'aventure. Actes du colloque Chemins d'accès: l'autonomisation, une école du visiteur ?. Paris : BNF. Consulté sur http://classes.bnf.fr/actes/6/ vandermeersch.pdf.

Viel, A. (2002). La valeur avalée. Dans M-O. Gonseth, J. Hainard et R. Kaehr (Dir.). Le musée cannibale (pp. 213-233). Neuchâtel : Musée d'Ethnographie de Neuchâtel.

\section{NOTES}

1. Les premiers cabinets de curiosités proposaient l'appréhension et la connaissance de l'univers à travers l'inventaire hétéroclite et exhaustif des choses rares, singulières et représentatives. Les naturalias renvoyaient alors aux productions du règne naturel; les artificialias aux créations humaines ; les scientificas aux instruments de mesures scientifiques; les exoticas aux objets extraeuropéens; et les mirabelias aux productions exceptionnelles et merveilleuses. Cependant ces ontologies sont rendues inopérantes par leur caractère poreux. Ainsi un animal peut-il relever de plusieurs classifications puisque son organisme est une création de la nature ; sa naturalisation une modification humaine, à usage scientifique et/ou artistique; et son origine peut dépendre de n'importe quel continent. Quant au terme musealia (anglais : exhibit), il s'agit d'un néologisme latinisant qui renvoie aux objets décontextualisés, conservés et/ou exposés dans des musées, peu importe leur ontologie primaire. L'usage et la présence muséales expliquent principalement le rattachement d'une chose à cette ontologie.

2. Industriel ardennais et chasseur naturaliste responsable.

3. Journaliste et photographe, elle initie François Sommer à la photographie animalière. Ils fondent ensemble l'Association française de Chasse Photographique, dans l'optique de prolonger la rencontre éphémère avec l'animal en rendant la mise à mort contingente.

4. « Fondation François Sommer, pour la chasse et la nature » depuis 2014 ; elle est instituée le 21 décembre 1964 et reconnue d'utilité publique par décret du 30 novembre 1966.

5. 26 mai 1973. Aujourd'hui «École et domaine de Belval » elle accueille des résidences artistiques en lien avec le MCN, des stages cynégétiques (éthologie, balistique, sylviculture) et réalise des études scientifiques. 
6. Dans son discours inaugural, André Malraux déclara que le musée apportait le cadre «d'un rêve millénaire des hommes, de tout ce que la Terre leur apporte de communion, de vie ou de mort par la nature et la chasse. »

7. La même année le MCN devient Musée de France.

8. Sur les conseils de leur ami Georges de Lastic (collectionneur et conservateur, historien de l'art), les Sommer acquièrent des oeuvres d'art cynégétique, afin d'assoir et légitimer le statut muséal du MCN, ce qui conférera au musée son esthétique de "cabinet d'amateur» ou de chasseur gentilhomme.

9. Les objets ethnographiques relatifs à la chasse vont de l'armement à l'art décoratif cynégétiques.

10. L'objet canalisant le plus le souvenir de chasse étant le trophée, on le retrouvera (sur)exposé dans chacune des salles. Les trophées personnels des fondateurs entrent alors en collection dès la création du musée (passant directement du salon à la cimaise), ainsi que ceux de leurs proches, émanant de dons. Seuls quelques rares spécimens sont achetés ou commandés afin de compléter une collection.

11. François Sommer, passionné d'aviation, avait coutume de partir chasser en Afrique. Il en rapporta divers trophées, ainsi que des armes et des objets zoomorphes.

12. Confere Vincent Leblanc, Julien Bondaz ou Maxime Michaud sur le rôle des campagnes cynégétiques lors des expéditions scientifiques des $\mathrm{XIX}^{\text {ème }}$ et $\mathrm{XX}^{\text {ème }}$ siècles dont les artefacts peuplent largement les musées ethnographiques et les muséums d'histoire naturelle.

13. Réclamant l'interdiction des activités cynégétiques, les mouvements anti-chasse prennent de l'ampleur depuis la fin du XX⿳亠̀⿵冂丶 ${ }^{2}$ siècle. Des manifestes sont publiés, des pétitions sont signées et des chasses sont perturbées (manifestants qui s'interposent entre le gibier et le chasseur, meutes de vénerie lancées sur de mauvaises voies et parfois coups et insultes fusent). «Pro » et "anti » chasse politisent (droite versus gauche), dualisent (citadins versus ruraux) et se disputent la paternité de l'écologie, de l'éthologie et in fine de la gestion (voire de la possession) de la nature.

14. L'animal favori de François Sommer.

15. Taxidermie de l'animal en entier, représentant un spécimen en position de vie.

16. À l'instar de la «Salle du Cerf et du Loup » dont le menuisement de chêne ancre davantage l'allégorie boisée.

17. Taxidermie de la tête de l'animal, montée sur un socle en bois.

18. Héritage des studiolo où les oeuvres, et parfois les typologies d'objets, se mélangeaient afin que le collectionneur puisse jouir d'un seul regard l'ensemble de sa collection, et éventuellement la faire découvrir à autrui ; il est l'ancêtre muséographique du cabinet de curiosité qui intègre des naturalia.

19. Cela aboutit à la circulaire de 1977 qui donne naissance à l'éducation à l'environnement.

20. L'EDD connait ensuite des évolutions entre 2007 et 2013, puis en 2015 lors de la COP 21 pour inclure de nouvelles démarches éducatives et participatives.

21. Synonyme de «fumées » : excréments du cerf.

22. Empreinte(s) du pied du sanglier en vénerie, ou du gibier en chasse à tir.

23. Ils animent par exemple «Sus Scorfa Albinos» le trophée d'un sanglier albinos articulé, de l'artiste Nicolas Darrot.

24. Entretien avec le Secrétaire général du MCN.

25. Scolaires et individuels confondus - Rapport d'activité de la Fondation, 2013.

26. Parmi les oeuvres choisies figurent le bas-relief en bronze de Saint Cemin (2006) qui propose une allégorie de la nature et un voyage onirique dans la forêt ; "Méléagre tuant le sanglier de Calydon » de Theodor Boeyermans (1677) qui fait référence à un récit mythologique et à la place de la chasse dès l'Antiquité dans la genèse de notre société ; la tapisserie de Diane et Actéon (XVI ${ }^{\text {ème }}$ siècle) qui illustre Les Métamorphoses d'Ovide et la chasse dans les arts ; ou bien bien la 
corne de narval (présentée comme une corne de licorne) qui montre l'accroissement de la connaissance grâce aux sciences et aux collections.

27. Confere André Desvallées.

28. Confere Edward O. Wilson.

29. Confere Rapport Brundtland.

30. Dans le monumental « Hallali du cerf » (D.882.2.1, RF 327 - Musée d'Orsay) de Gustave Courbet peint en 1867 , ce sont les chiens qui attaquent le cerf tandis que le fouet du piqueur claque dans les airs. Il retient même l'un des chiens, pendant que le veneur est encore à cheval.

\section{RÉSUMÉS}

Kitsch ou de mauvais goût il y a encore une dizaine d'années, la taxidermie s'impose désormais comme un objet muséographique, scientifique et décoratif légitime. On visite volontiers un muséum d'histoire naturelle pour ses taxidermies, on chine les animaux empaillés mis au rebut et on décore son chez-soi avec des trophées géométriques ou en peluche. Bref, la taxidermie a le vent en poupe (Dalla Bernardina, 2013).

Profitant ou participant de ce retour de l'animal naturalisé et du trophée de chasse, les musées cynégétiques repensent la muséographie d'animaux dont l'origine (muséale) ne laisse aucun doute : la mise à mort. Aussi, à l'heure où les problématiques sont éco-logistes et animalistes, convient-il de questionner la pertinence de la monstration d'une dépouille animale a fortiori pour sensibiliser à l'environnement.

Afin de réfléchir à cela, nous partirons des trophées du Musée de la Chasse et de la Nature pour étudier le paradoxe muséographique que représente l'exhibition d'un animal chassé pour en faire un hymne à la vie.

Ten years ago taxidermy was seen as kitsch or off color, but is now a legitimate museographic, scientific and decorativ artefact. We gladly visit a natural history mu-seum for its taxidermies, we are looking for discarded stuffed animals and we decorate our homes with geometric or fake stuffed trophies. Hence, taxidermy is on the rise.

Making the most of stuffed animal fashion, hunting museums rethinking their museo-graphy to please visitors and show trophies whose exhibit is obvious : death. Further-more, at a time when ecology and animal rights are current issues, it is appropriate to think about the relevance of the demonstration of animal remains to raise awareness of the environment.

By examining hunting trophies from the parisian Musée de la Chasse et de la Nature, we explore how a museum use hunted animals to tell stories of life.

\section{INDEX}

Keywords : anthropology, museology, hunting museum, taxidermy, hunting trophy.

Mots-clés : anthropologie, muséologie, musée cynégétique, taxidermie, trophée de chasse. 


\section{AUTEUR}

\section{ANTOINE JEANNE}

Antoine Jeanne est doctorant en ethnologie à l'Université de Paris (ED 180 - Canthel EA 4545). Il est titulaire d'une allocation de recherche de Troisième cycle de l'École du Louvre où il suit un cursus en muséologie. À la croisée de deux disciplines, il étudie la chaîne opératoire de la patrimonialisation de l'animal (chassé) ainsi que les logiques expographiques des spécimens trophéisés. Il a notamment mené le récolement de la collection de trophées du Musée de la Chasse et de la Nature. (Courriel : antoinejeanne[@]outlook.com) 